\title{
Idiopathic Sudden Sensorineural Hearing Loss in Patients with Obstructive Sleep Apnea
}

\author{
Chin-Kuo Chen ${ }^{1,2}$ \\ Shih Chieh Shen ${ }^{1-3}$ \\ Li-Ang Lee (iD) ${ }^{1,2}$ \\ Ming-Hui Sun ${ }^{2,4}$ \\ Ning-Hung Chen (iD ${ }^{2,5}$ \\ Li-Pang Chuang (D) ${ }^{2,5}$ \\ Hsueh-Yu Li (iD) ${ }^{1,2}$ \\ 'Department of Otolaryngology-Head \\ and Neck Surgery, Sleep Center, Chang \\ Gung Memorial Hospital (Linkou), \\ Taoyuan City, Taiwan; ${ }^{2}$ College of \\ Medicine, Chang Gung University, \\ Taoyuan City, Taiwan; ${ }^{3}$ Department of \\ Otolaryngology-Head and Neck Surgery, \\ New Taipei Municipal Tucheng Hospital, \\ New Taipei City, Taiwan; ${ }^{4}$ Department of \\ Ophthalmology, Chang Gung Memorial \\ Hospital (Linkou), Taoyuan City, Taiwan; \\ ${ }^{5}$ Department of Pulmonary and Critical \\ Care Medicine, Sleep Center, Chang \\ Gung Memorial Hospital (Linkou), \\ Taoyuan City, Taiwan
}

Correspondence: Hsueh-Yu Li; Shih Chieh Shen

Department of Otolaryngology-Head and Neck Surgery, Sleep Center, Chang Gung Memorial Hospital (Linkou), 33, No. 5, Fushing Street, Gueishan Shiang, Taoyuan City, Taiwan

Tel +886-3-328I 200 ext.3966

Fax +886-3-397936।

Email hyli38@cgmh.org.tw;

b9102083@cgmh.org.tw
Purpose: Obstructive sleep apnea (OSA) is characterized by recurring hypoxic-apneic events during sleep, and labyrinthine vascular compromise is a pathophysiologic hallmark of idiopathic sudden sensorineural hearing loss (ISSNHL). Some reports have discussed the relationship between OSA and hearing impairment; however, few have examined hearing prognosis in OSA and patients without OSA with ISSNHL. We aimed to investigate clinical manifestations of ISSNHL in patients with OSA, including severity of hearing loss and response to treatment.

Patients and Methods: A case-control study was conducted by extracting data from the sleep center and cochlea center databases of the Chang Gung Memorial Hospital. A retrospective chart review was performed to include confirmed adult OSA patients diagnosed with unilateral ISSNHL. Age and sex-matched patients without OSA with ISSNHL were enrolled as controls. Pure-tone average (PTA) thresholds were measured at specific frequencies. Changes in PTA before and after standard treatment with oral prednisolone ( $1 \mathrm{mg} / \mathrm{kg} /$ day for 5 days, then tapered) and between participants with OSA and without OSA were compared. Standard treatment was given to all ISSNHL patients.

Results: Twenty-eight out of $8500(0.33 \%)$ OSA patients experienced subsequent ISSNHL in 9 years. Patients with OSA $(n=28)$ had poorer high-frequency perception in the unaffected ear than the patients without OSA $(n=120)$, although the difference was not significant. Hearing in the affected ear among patients with OSA was comparable to that patients without OSA at individual frequencies and average, suggesting no difference in hearing loss in the affected ear between the two groups. In terms of high-frequencies $(4000$ and $8000 \mathrm{~Hz})$ perception, patients with OSA had significantly poorer responses to steroid treatment than patients without OSA.

Conclusion: ISSNHL may be one of the auditory complications associated with OSA. Patients with OSA had poorer prednisolone related hearing improvement in high frequencies than patients without OSA. Despite study limitations, OSA-related hypoxia and snoring noise is hazardous to hearing and standard treatments with CPAP is suggested in OSA patients for both holistic and auditory health.

Keywords: obstructive sleep apnea, idiopathic sudden sensorineural hearing loss, highfrequency, hypoxemia, pure-tone average, frequencies

\section{Introduction}

Obstructive sleep apnea (OSA), characterized by recurring events of upper airway collapse during sleep, affects nearly 1 billion people worldwide. ${ }^{1,2}$ Apnea and hypopnea are the hallmarks of OSA contributing to physiological instabilities, which include excessive fluctuations in intrathoracic and intracranial pressure, changes in the heart rate, and eventually, tissue desaturation. ${ }^{3,4}$ The pathogenesis 
of recurrent hypoxia/hypercapnia and chronic inflammatory status in OSA is associated with cardiovascular diseases and neurocognitive deficits. ${ }^{3-6}$ Moreover, recurrent hypoxia and noise exposure to the cochlea causes marked hearing dysfunction. ${ }^{7-9}$

Organs without communication branch vesicles, such as the inner ear system, are easily influenced by recurrent hypoxia and inflammation. However, idiopathic sudden sensorineural hearing loss (ISSNHL) is an acute condition that presents with sudden deafness of $\geq 30 \mathrm{~dB}$ in at least three contiguous frequencies in $<3$ days. ISSNHL has an incidence of 5-27 cases per 100,000 per year. ${ }^{10,11}$ Although uncertain causes need to be excluded and the origin remains unclear, the main pathophysiological points linked to ISSNHL include labyrinthine vascular compromise, labyrinthine viral infection, intracochlear membrane rupture, and immune-mediated inner ear disease. ${ }^{12}$

Prolonged hypoxia can elicit a chronic inflammatory status and excessive oxidative stress, potentially compromising the integrity of the inner ear's microvasculature in patients with OSA. ${ }^{13}$ Moreover, patients with OSA may have worsening hearing status and impaired high-frequency sound perception. ${ }^{8,14,15}$ Furthermore, a population-based case-control study has indicated that male patients with ISSNHL often have a history of OSA. ${ }^{16}$ Nonetheless, there remains a paucity of information on the clinical manifestations of ISSNHL in patients with OSA.

In this study, we aimed to investigate the clinical manifestations of ISSNHL in patients with OSA, including severity of hearing loss and response to treatment.

\section{Materials and Methods}

\section{Data Sources and Study Population}

A retrospective chart review was conducted by extracting data from the sleep center and cochlear center at Chang Gung Memorial Hospital from 2009 to 2017. The Joint Institutional Review Board of Chang Gung Memorial Hospital approved (No. 201601393B0C601) the study, which was performed according to the principles outlined in the Declaration of Helsinki. Participants' informed consent was waived due to retrospective chart review in this study. Adult patients diagnosed with unilateral ISSNHL were included, and the exclusion criteria comprised insomnia, simple snoring, preexisting otologic or neurologic disorders, prior otologic procedures, and comorbidities of the liver and the endocrine system. Also, all ISSNHL patients were diagnosed in the cochlear center after a thorough protocol including history taking, physical examination, otoscopy, audiometric tests, stapedius reflex, vestibular examination, tympanometry, bloody tests, ABR, MRI and/or CT scan to exclude its cause from infection, barotrauma, ototoxic drug, sickle cell disease, acoustic neuroma, etc. At the sleep center, 8500 OSA patients were recruited, and 28 of those who consequently developed ISSNHL were enrolled in this study. On the other hand, patients with ISSNHL were acquired from the cochlear center. Since sleep disorders were associated with inner ear disease, patients with ISSNHL were excluded if they had sleep-related symptoms/conditions including insomnia, sleep-deprivation, shift work, snoring, witnessed sleep apnea, or daytime sleepiness/fatigue based on the structured chart review. Furthermore, stratified sampling was applied to select patients without OSA group according to the average age of the OSA group (44-64 years), and the ratio of male to female (3:1); simple random sampling was used to select 85 men and 35 women, with a total of 120 patients. Overall, we enrolled 28 patients with OSA and 120 patients without OSA, matched by age and sex.

\section{Study Outcome and Measurements}

All ISSNHL patients were administered oral prednisolone (1 $\mathrm{mg} / \mathrm{kg} /$ day) for 5 days, followed by tapering within 5 days. Patients who failed systemic steroid therapy within 14 days were given intratympanic injection of $0.4 \mathrm{~mL}$ dexamethasone and/or HBO therapy. Audiometric tests were performed at specific frequencies of 250, 500, $1000,2000,4000$, and $8000 \mathrm{~Hz}$ before and after the oral prednisolone treatment. Pure-tone average (PTA), the average of hearing thresholds at 500, 1000, 2000, and $4000 \mathrm{~Hz}$, was calculated. Hearing loss was defined as the threshold difference between the affected and unaffected ears, and hearing improvement was defined as the threshold difference before and after the treatment. OSA was diagnosed based on polysomnography. The apnea-hypopnea index (AHI) was defined as the average number of episodes of apneas and hypopneas per hour of sleep. The participants were categorized into two groups: OSA and without OSA groups. Patients with AHI $>5$ were categorized as the OSA group.

\section{Polysomnography}

All patients with OSA underwent full-night polysomnography at the sleep center of the Chang Gung Memorial Hospital. The patients avoided daytime naps and consumption of strong tea, coffee, alcohol, or sedative drugs. The sleep monitor assay was performed on the night of admission to analyze sleep for approximately $6-8 \mathrm{~h}$. 
Rechtschaffen and Kales have rated sleep stages according to the guidelines. ${ }^{17}$ Standard polysomnography was performed to document sleep parameters. Arousals were defined according to the American Sleep Disorders Association criteria. ${ }^{18}$ In adults, apnea is defined as $>90 \%$ reduction of flow for $>10 \mathrm{~s}$ and classified as obstructive in the presence of rib cage and abdominal wall movements, or central with a lack of respiratory effort. Hypopneas are defined as $>50 \%$ reduction in flow for at least two breaths with subsequent oxygen desaturation of at least $3 \%$ or arousal. ${ }^{19}$

\section{Sample Size}

The sample size was determined using $\mathrm{G}^{*}$ power version $3.1^{20}$ and the results of a previous analysis with this effect size revealed that we needed a sample size, $\mathrm{N}=20$, to achieve $95 \%$ power at $\alpha=0.05$.

\section{Statistical Analysis}

Statistical analysis was performed using the Statistical Package for Social Science software (version 22.0; IBM Corp., Armonk, IBM Company, USA). Continuous variables are expressed as means and standard deviations (SD), and categorical variables are expressed as counts and frequencies. Effect sizes with 95\% confidence intervals (CIs) were calculated for the variables. Independent samples t-tests were used to compare age, sex, and pure-tone audiometry between the OSA and without OSA groups. A paired sample $t$-test was used to compare the preoperative and postoperative data. The Wilcoxon signed-rank test was used to compare the preoperative and postoperative data of a small sample. Statistical significance was set at $P<0.05$.

\section{Results}

\section{Incidence of ISSNHL in OSA}

According to our data, 28 of $8500(0.33 \%)$ patients with OSA experienced subsequent ISSNHL.

\section{Patient Characteristics}

Twenty-eight OSA patients $(21 \mathrm{men})$ and 120 patients without OSA ( $85 \mathrm{men}$ ) were enrolled in the study. There were no significant differences in demographic variables, such as age, sex, presence or absence of vertigo, type of audiogram, initial treatment days after onset of hearing loss, baseline hearing of the unaffected ear, and comorbidities (diabetes mellitus, hypertension, renal disease, and coronary artery diseases) between the OSA and without OSA groups. (Table 1).

\section{Hearing Presentation of Unaffected Ears in the OSA and Without OSA Groups}

The baseline PTA of the unaffected ear in the OSA and without OSA groups were $24.6 \pm 12.9 \mathrm{~dB}$ and $23.9 \pm 16.0$ $\mathrm{dB}$ (Cohen's d, 0.05; 95\% CI $[-6.50,-5.27] ; P=0.83$ ), respectively (Table 2 ).

\section{Hearing Presentation of the Affected Ears in the OSA and Without OSA Groups}

The initial PTA of the affected ears in the OSA and without OSA groups were $63.5 \pm 18.9 \mathrm{~dB}$ and $61.2 \pm 20.4 \mathrm{~dB}$ (Cohen's d, 0.10; 95\% CI $[-6.33,-10.15] ; P=0.59$ ), respectively (Table 2). The affected ears in both groups had comparable hearing presentations.

Table I Demographics and Clinical Characteristics of ISSNHL in patients with OSA and without OSA

\begin{tabular}{|l|l|l|l|l|}
\hline & Without OSA (n= 1 20) & OSA (n=28) & Effect Size/OR (95\% CI) & P value \\
\hline Age, mean (SD), y & $52.9(8.4)$ & $53.9(9.7)$ & $0.11(-4.57-2.59)$ & 0.587 \\
\hline $\begin{array}{l}\text { Gender, n (\%) } \\
\text { Male }\end{array}$ & $85(70.8)$ & $21(75.0)$ & $0.94(0.74-1.20)$ & 0.817 \\
Female & $35(29.2)$ & $7(25.0)$ & $1.17(0.58-2.35)$ & \\
\hline $\begin{array}{l}\text { Comorbidity } \\
\text { DM, n (\%) }\end{array}$ & $31(25.8)$ & $9(32.1)$ & $0.80(0.43-1.49)$ & 0.637 \\
HTN, n (\%) & $32(26.7)$ & $12(42.9)$ & $0.62(0.37-1.05)$ & 0.110 \\
CAD, n (\%) & $6(5.0)$ & $2(7.1)$ & $0.70(0.15-3.29)$ & 1.000 \\
\hline
\end{tabular}

Notes: Average $=(500+1 k+2 k+4 k) / 4$. ${ }^{a}$ For continuous variables, effect size is the median of the difference of medians between the groups, and the $95 \% \mathrm{Cl}$ around that difference was calculated using the method of Cohen's $d$ for $t$ test. For categorical variables, effect size is the difference between proportions of the groups with relative $95 \%$ Cls calculated around that difference.

Abbreviations: OSA, obstructive sleep apnea; OR, odds ratio; $\mathrm{Cl}$, confidence intervals; SD, standard deviation; dB, decibel; DM, diabetes mellitus; HTN, hypertension; CAD, coronary artery disease. 
Table 2 The Hearing Thresholds of the Idiopathic Sudden Sensorineural Hearing Loss Ear in Patients with Obstructive Sleep Apnea (OSA) and Patients Without OSA, Before Treatment

\begin{tabular}{|c|c|c|c|c|}
\hline & Without OSA $(n=\mid 20)$ & OSA $(n=28)$ & Effect Size $(95 \% \mathrm{Cl})^{\mathrm{a}}$ & $P$ value \\
\hline \multicolumn{5}{|c|}{ Baseline hearing of the unaffected ear (in Decibels), mean (SD) } \\
\hline 250 & $19.5(12.8)$ & $18.6(10.0)$ & $0.04(-4.47,-5.50)$ & 0.709 \\
\hline 500 & $20.0(14.6)$ & $18.0(9.6)$ & $0.13(-3.96,-6.97)$ & 0.500 \\
\hline Ik & $21.3(16.9)$ & $20.2(12.9)$ & $0.06(-5.10,-6.99)$ & 0.744 \\
\hline $2 k$ & $22.0(18.1)$ & $22.7(16.9)$ & $0.05(-7.59,-5.98)$ & 0.865 \\
\hline $4 \mathrm{k}$ & $32.1(21.7)$ & $37.3(21.9)$ & $0.19(-12.91,-4.68)$ & 0.256 \\
\hline $8 \mathrm{k}$ & $38.4(25.4)$ & $43.6(23.1)$ & $0.21(-14.90,-5.01)$ & 0.326 \\
\hline Average & $23.9(16.0)$ & $24.6(12.9)$ & $0.05(-6.50,-5.27)$ & 0.833 \\
\hline \multicolumn{5}{|c|}{ Hearing of the affected ear (in Decibels), mean (SD) } \\
\hline 250 & $58.3(20.0)$ & $52.1(20.5)$ & $0.54(2.5 \mathrm{I},-18.70)$ & 0.145 \\
\hline 500 & $61.3(21.0)$ & $62.0(19.3)$ & $0.14(-5.54,-11.19)$ & 0.885 \\
\hline Ik & $62.2(22.2)$ & $66.4(21.1)$ & $0.04(-9.82,-7.88)$ & 0.362 \\
\hline $2 k$ & $57.4(23.7)$ & $60.0(24.2)$ & $0.06(-8.04,-11.04)$ & 0.600 \\
\hline $4 k$ & $63.3(23.3)$ & $64.8(23.5)$ & $0.18(-5.66,-14.27)$ & 0.755 \\
\hline $8 k$ & $68.1(23.5)$ & $67.1(28.8)$ & $0.23(-4.30,-16.26)$ & 0.855 \\
\hline Average & $61.2(20.4)$ & $63.5(18.9)$ & $0.10(-6.33,-10.15)$ & 0.594 \\
\hline \multicolumn{5}{|c|}{ Hearing loss of the affected ear (in Decibels), mean (SD) } \\
\hline 250 & $38.8(20.0)$ & $33.6(20.0)$ & $0.51(1.97,-18.22)$ & 0.215 \\
\hline 500 & $41.3(22.4)$ & $43.9(21.4)$ & $0.06(-7.73,-10.37)$ & 0.578 \\
\hline Ik & $40.9(24.6)$ & $46.3(21.5)$ & $0.09(-11.53,-7.69)$ & 0.292 \\
\hline $2 k$ & $35.3(25.7)$ & $37.3(25.2)$ & $0.09(-8.03,-12.64)$ & 0.712 \\
\hline $4 k$ & $31.2(25.8)$ & $27.5(24.4)$ & $0.33(-2.21,-19.04)$ & 0.495 \\
\hline $8 \mathrm{k}$ & $29.7(26.9)$ & $23.6(25.3)$ & $0.42(-0.19,-22.04)$ & 0.276 \\
\hline Average & $37.4(22.3)$ & $38.9(19.5)$ & $0.12(-6.32,-11.48)$ & 0.744 \\
\hline
\end{tabular}

Notes: Average $=(500+1 k+2 k+4 k) / 4$. ${ }^{a}$ For continuous variables, the effect size is the median of the difference of medians between the groups, and the $95 \% \mathrm{Cl}$ around that difference was calculated using Cohen's $d$ for the $t$-test.

Abbreviations: $\mathrm{Cl}$, confidence intervals; SD, standard deviation.

\section{Initial Hearing Loss of the Affected Ear in the OSA and Without OSA Groups}

Before treatment, the hearing loss of PTA in the OSA and without OSA groups were $38.9 \pm 19.5 \mathrm{~dB}$ and $37.4 \pm 22.3$ dB (Cohen's d, 0.12; 95\% CI $[-6.32,-11.48] ; P=0.74$ ), respectively (Table 2 ).

\section{Hearing Presentation of the Affected Ears After Treatments in the OSA and Without OSA Groups}

After treatment, the OSA group had less hearing improvement at all frequencies than the without OSA group. The without OSA group had significant threshold improvements in frequencies at 2000, 4000 and $8000 \mathrm{~Hz}$ than the OSA group $(P<0.05$; Table 3$)$.

\section{Hearing Loss of the Affected Ears After Treatments in Both Groups}

Both groups had comparable hearing loss after treatment $(\mathrm{P}>0.05)$; however, the OSA group had a tendency toward more hearing loss than the without OSA group. Moreover, the OSA group had more hearing loss of PTA after treatment than the without OSA group (Cohen's d, 0.04; 95\% CI $[-8.08,-6.30] ; P=0.018$; Table 3 ).

\section{Hearing Presentation Response to Treatments of ISSNHL in the OSA and Without OSA Groups}

The without OSA group showed significant hearing improvements at all frequencies $(250-8000 \mathrm{~Hz} ; P<0.05$; Figure 1A). In the OSA group, the hearing sensitivity 
Table 3 The Hearing Thresholds of the Idiopathic Sudden Sensorineural Hearing Loss Ear in Patients with Obstructive Sleep Apnea (OSA) and Patients Without OSA, After Treatment

\begin{tabular}{|c|c|c|c|c|}
\hline & Without OSA $(n=120)$ & OSA $(n=28)$ & Effect Size $(95 \% \mathrm{CI})^{\mathrm{a}}$ & $P$ value \\
\hline \multicolumn{5}{|c|}{ Hearing of the affected ear (in Decibels), mean (SD) } \\
\hline 250 & $32.2(19.8)$ & $33.9(21.1)$ & $0.26(-3.79,-15.10)$ & 0.684 \\
\hline 500 & $33.9(21.7)$ & $40.0(22.3)$ & $0.06(-8.31,-11.15)$ & 0.182 \\
\hline Ik & $36.8(22.5)$ & $45.4(22.2)$ & $0.05(-11.33,-9.28)$ & 0.070 \\
\hline $2 k$ & $37.9(23.1)$ & $48.9(20.7)$ & $0.13(-13.26,-6.98)$ & $0.022^{*}$ \\
\hline $4 k$ & $47.4(24.1)$ & $58.6(23.3)$ & $0.13(-14.28,-7.63)$ & $0.028^{*}$ \\
\hline $8 \mathrm{k}$ & $53.8(26.1)$ & $67.1(26.4)$ & $0.25(-18.17,-4.72)$ & $0.016^{*}$ \\
\hline Average & $39.0(20.3)$ & $48.2(17.4)$ & $0.07(-9.35,-6.31)$ & $0.028^{*}$ \\
\hline \multicolumn{5}{|c|}{ Hearing loss of the affected ear (in Decibels), mean (SD) } \\
\hline 250 & $12.7(2 \mid .8)$ & $15.4(20.5)$ & $0.23(-4.66,-14.95)$ & 0.553 \\
\hline 500 & $13.9(22.1)$ & $22.0(19.4)$ & $0.00(-8.70,-8.52)$ & 0.077 \\
\hline Ik & $15.5(24.6)$ & $25.2(19.4)$ & $0.09(-10.73,-6.79)$ & 0.053 \\
\hline $2 k$ & $15.9(26.8)$ & $26.3(19.3)$ & $0.10(-11.19,-6.53)$ & 0.055 \\
\hline $4 \mathrm{k}$ & I5.3 (26.8) & $21.3(22.6)$ & $0.03(-10.40,-11.98)$ & 0.275 \\
\hline $8 \mathrm{k}$ & I5.4 (29.1) & $23.6(24.5)$ & $0.07(-13.44,-9.88)$ & 0.169 \\
\hline Average & $15.2(23.0)$ & $23.8(15.0)$ & $0.04(-8.08,-6.30)$ & $0.018^{*}$ \\
\hline
\end{tabular}

Notes: Average $=(500+1 k+2 k+4 k) / 4 . * P<0.05$, statistically significant differences between the groups. ${ }^{\mathrm{a}}$ For continuous variables, the effect size is the median of the difference of medians between the groups, and the $95 \% \mathrm{Cl}$ around that difference was calculated using Cohen's $d$ for the $t$-test.

Abbreviations: OSA, obstructive sleep apnea; ISSNHL, idiopathic sudden sensorineural hearing loss; PTA, pure-tone average; AHI, apnea-hypopnea index; SD, standard deviations; $\mathrm{Cl}$, confidence interval.

significantly improved after treatment at all frequencies, except at $8000 \mathrm{~Hz}$ (Figure 1B). Both groups also showed a significant improvement in PTA after treatment.

\section{Comparison of the Extent of Hearing Improvement in Response to Treatments in OSA and Without OSA Groups}

Both groups showed improvements at all hearing frequencies. Notably, the OSA group had significantly less hearing improvement at frequencies of 4000 and $8000 \mathrm{~Hz}$ than those in the without OSA group (Figure 2).

\section{Discussion}

This study is the first to investigate the clinical manifestations of ISSNHL in patients with OSA before and after treatment, and compare them with patients without OSA as controls. This study benefited from data acquisition based on a single institutional setting, ensuring that all enrolled patients followed standardized diagnostics and therapeutics for ISSNHL. The clinical presentation of ISSNHL in patients with OSA and the linkage of OSA to ISSNHL were addressed from different perspectives: 1) the pathophysiological plausibility and experimental evidence, and 2) coherence to observational studies and biological gradients.

OSA with neuro-otologic disease risk has been reported on by several studies; ${ }^{21-25}$ however, the causal relationship between OSA and ISSNHL remains unclear. A populationbased case-control study reported that patients with ISSNHL have an association with OSA. ${ }^{16}$ In a crosssectional study, patients with ISSNHL $(n=27)$ had OSA more frequently than those with normal hearing $(n=33) .{ }^{26}$ Our study showed that 28 out of $8500(0.33 \%)$ patients with OSA (aged 20 to 65 years old) experienced ISSNHL over 9 years. In two epidemiological studies, ${ }^{10,11}$ the prevalence of ISSNHL in the general population aged 18 to 64 years old was 9.8 (Taiwanese, Asian) and 27 (American, Caucasian) per 100,000 population. Although studies were not entirely comparable and the causal inference relationship could not be established, our study revealed a potentially high incidence of ISSNHL in OSA patients. Therefore, the risk of ISSNHL should be taken into account for OSA patients, and the comorbid OSA in ISSNHL patients should be evaluated, vice versa.

OSA has a detrimental effect on the cochlea, which is particularly sensitive to circulatory alterations since it is supplied by a single terminal artery and lacks adequate 
A

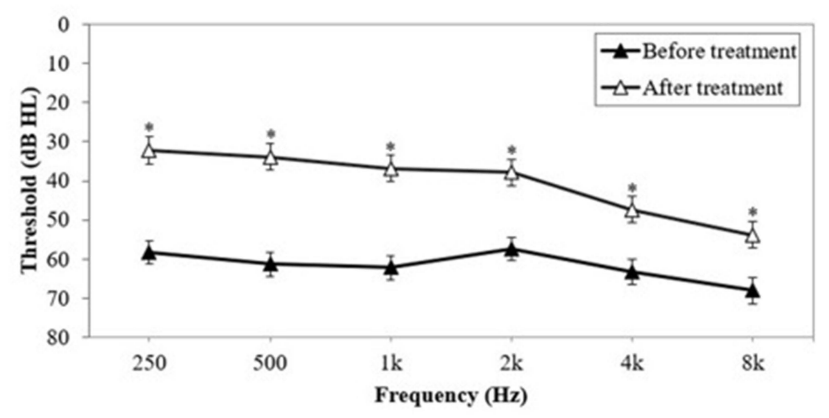

B

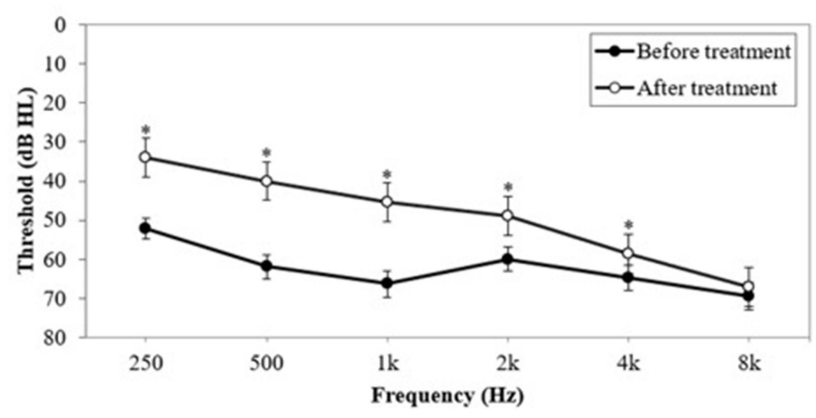

Figure I The hearing thresholds of the idiopathic sudden sensorineural hearing loss ear in patients with obstructive sleep apnea (OSA) (B) and patients without OSA (A) before and after treatment. $* P<0.05$; a statistically significant Wilcoxon signed-rank test; $b$ statistically significant paired $t$-test.

collateral blood supply. ${ }^{27}$ OSA is characterized by repeated upper airway obstruction and related hypoxia during sleep, directly resulting in decreased cerebral blood flow, elevated sympathetic nerve activity, and oscillations of blood pressure, which cause adverse cerebrovascular events and in turn, ischemic injury to the cochlea. ${ }^{16}$ Previous studies have emphasized that due to high metabolic demand, the inner ear is vulnerable to periodic hypoxia. ${ }^{28,29}$ Long-term exposure to the noisy snoring sound in OSA patients could damage the inner ear and incur hearing impairment. Clinical studies have revealed a decreased perception of high-frequency sounds $(4000$ and $8000 \mathrm{~Hz})$ in patients with moderateto-severe OSA. ${ }^{15,30}$ This study showed similar findings, and although not statistically significant, patients with OSA had a poorer high-frequency perception in the unaffected ear than patients without OSA. This suggests that OSA could be a risk factor for auditory dysfunction. ${ }^{31,32}$

Hearing of the affected ears in the OSA and patients without OSA were comparable at individual frequencies and the average of the frequencies, and did not indicate hearing loss differences in the affected ear between the two study groups. We presume that patients with OSA experience chronic repeated hypoxia, which becomes more resistant to hemodynamic changes from ISSNHL, and consequently, compensates for its hearing loss.

ISSNHL with OSA had a poorer response to treatment at a high-frequency. The hearing threshold of the affected ear improved after treatment (steroid therapy) at all individual frequencies in both groups, except at $8000 \mathrm{~Hz}$ in the

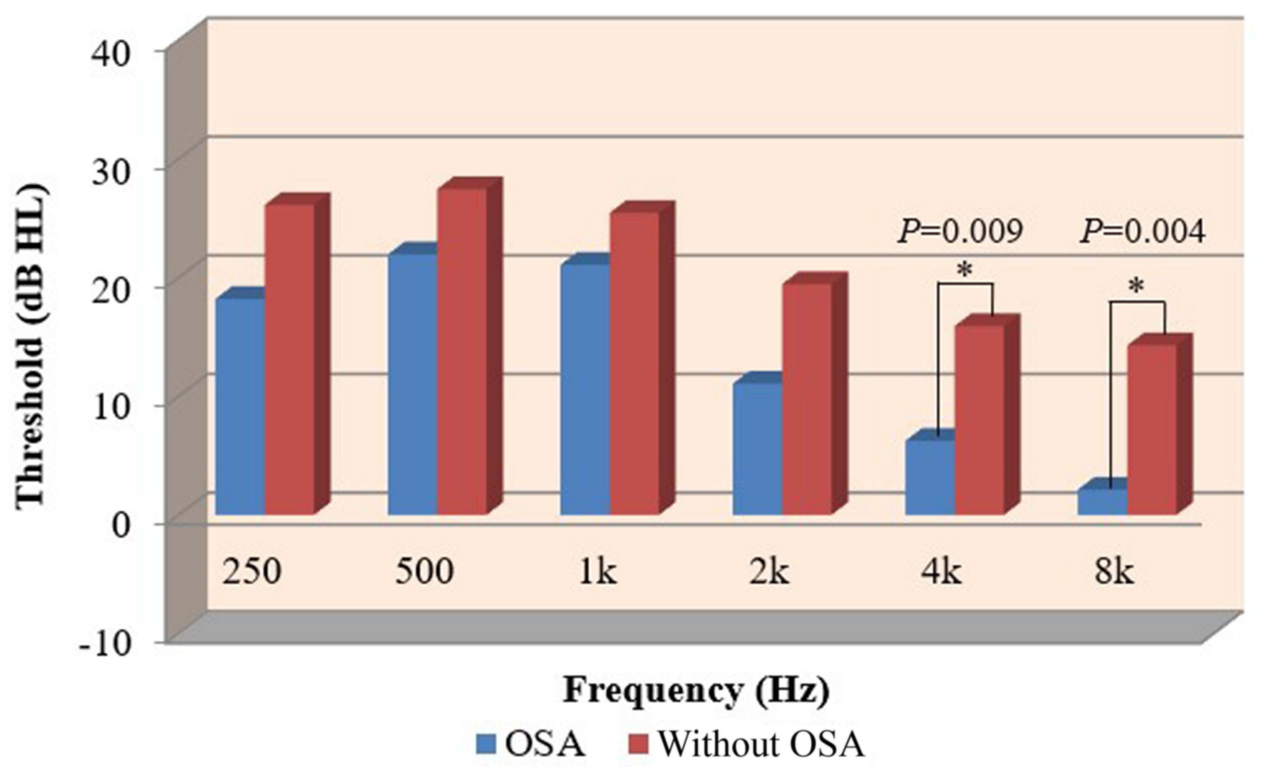

Figure 2 The magnitude of hearing improvements in patients with obstructive sleep apnea (OSA) and patients without OSA with idiopathic sudden sensorineural hearing loss. $* P<0.05$, statistically significant independent $t$-test. 


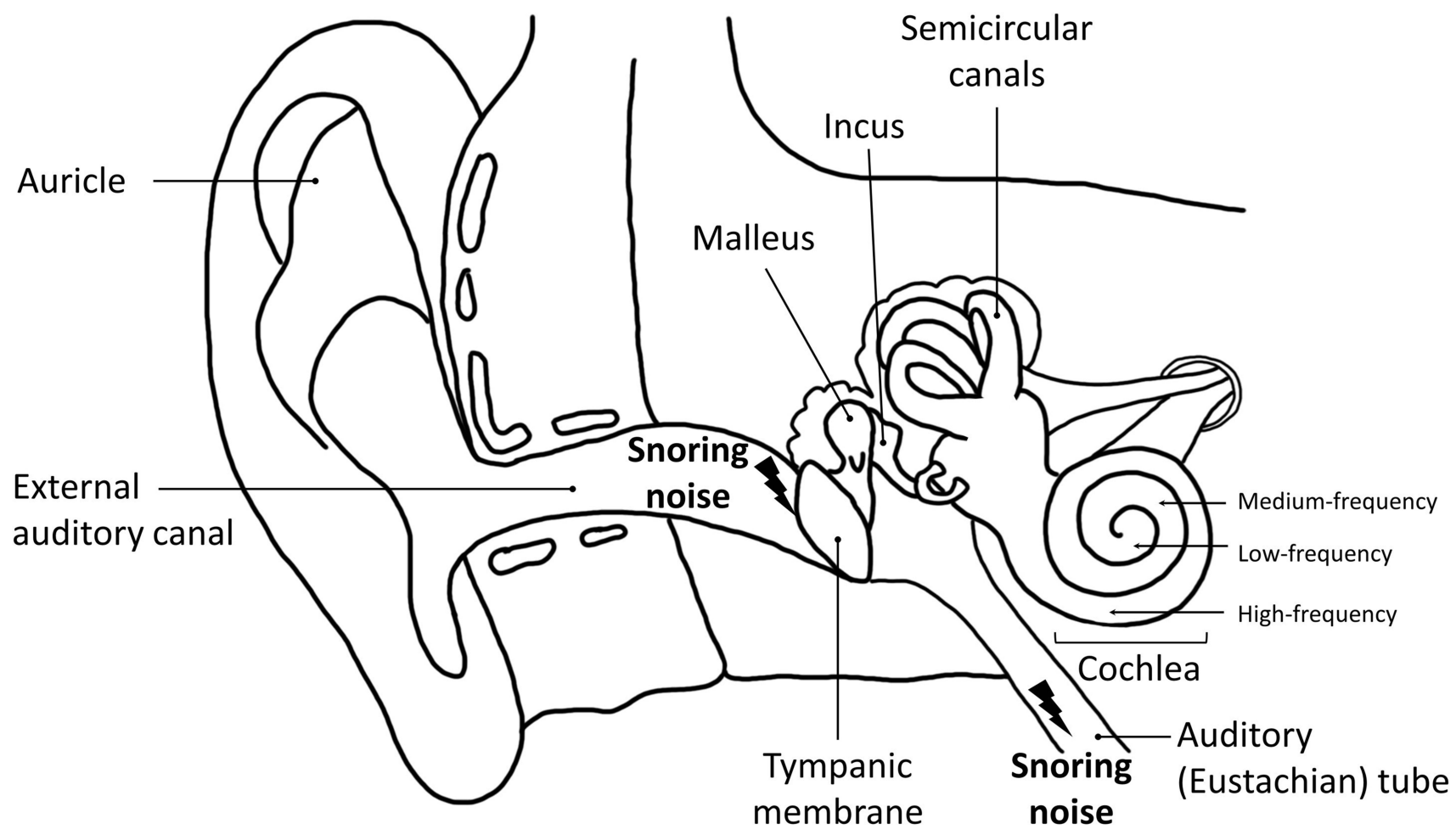

Figure 3 Different parts of the cochlea corresponding to different frequencies. High-frequency hearing in the cochlear basal turn is exposed and vulnerable to the snoring noise.

OSA group (Figure 1A and B). Research has revealed that OSA and ISSNHL are inflammation-related, and inflammatory cytokines are higher in patients with OSA. ${ }^{33}$ Since steroids are potent inflammatory inhibitors, it was not surprising to observe effective improvement in the hearing threshold of the affected ear after treatment. Notably, the improvement in hearing declined after $2000 \mathrm{~Hz}$, with no change at $8000 \mathrm{~Hz}$ in the patients with OSA (Figure 1B). Furthermore, a significantly poorer improvement in hearing was noted at 4000 and $8000 \mathrm{~Hz}$ in the OSA group than in the without OSA group (Figure 2). We speculated that noise exposure from snoring sounds may have played a role in the treatment outcome in patients with OSA. The repeated loud snoring sound transmits through the velopharyngeal channel (Eustachian tube) and conductive mechanism into the hum that causes acoustic trauma to the auditory organ, particularly the exterior part, which corresponds to high-frequency hearing (Figure 3 ). The persistent acoustic trauma from snoring may explain the poor response to treatment at a high-frequency in patients with OSA. In addition to acoustic trauma, chronic inflammation, and intermittent hypoxia could contribute to rendering OSA patients refractory to treatment during the insult of ISSNHL. The poorer hearing recovery in patients with
OSA also suggested that treatment of OSA is needed for both general and specific (auditory) health.

Our study has several limitations. This research could be biased by its limited sample size, lack of confounder stratification, selection of the study population, and the suboptimal method adopted in the matching process. The matching process in this study did not adopt some of the advanced matching procedures, such as propensity score matching. Reasons for utilizing a more traditional matching method was to ensure that bias would not be further introduced during the construction of regression models necessary for propensity score matching, while there were few potential confounders of sufficient evidence. On the other hand, due to the small sample size, it was less feasible to stratify within the OSA group based on the severity of OSA or the type of OSA treatment. Also, due to the disadvantages of the retrospective chart review, this study lacked detailed information regarding the efficiency of OSA treatment, compliance and adherence of continuous positive airway pressure, and the precise chronological relationship between the treatment and the onset of ISSNHL. As a result, it was less likely to evaluate the effects of OSA treatment on clinical manifestations of ISSNHL. Nevertheless, we aimed to present the preliminary findings for the relationship between OSA and ISSNHL. Studies of larger sample sizes and a robust 
study design will be needed to better understand the relationship between OSA and ISSNHL.

\section{Conclusion}

OSA may be associated with ISSNHL. The hearing thresholds of both unaffected and ISSNHL ears did not differ between the OSA and without OSA groups. Patients in the OSA group had poorer hearing improvement after treatment, especially at high frequencies (4000 and $8000 \mathrm{~Hz}$ ). Also, from the perspective of preventive medicine, it is crucial to timely intervene OSA patients with standard treatment of CPAP and sufficient support for both general and specific (auditory) health, and screening ISSNHL patients for the comorbid OSA should be considered.

\section{Acknowledgments}

The authors would like to thank Ms. Yen-Ting Chiang, Ms. Ling-Hsuan Chuang, and Ms. Shin-Fei Chi for their assistance in data collection and statistical analyses.

\section{Disclosure}

The authors report no conflicts of interest in this work. The authors disclosed receipt of the following financial support for the research, authorship, and/or publication of this article. This research project was supported by the Chang Gung Memorial Hospital (Linkou, Taiwan) under Grant Number CORPG3F0851 and CMRPG3J0701-3.

\section{References}

1. Benjafield AV, Ayas NT, Eastwood PR, et al. Estimation of the global prevalence and burden of obstructive sleep apnoea: a literature-based analysis. Lancet Respir Med. 2019;7(8):687-698. doi:10.1016/S22132600(19)30198-5

2. American Academy of Sleep Medicine. The International Classification of Sleep Disorders: Diagnostic and Coding Manual. 2nd ed. Westchester, IL: American Academy of Sleep Medicine; 2005:51-55.

3. Dewan NA, Nieto FJ, Somers VK. Intermittent hypoxemia and OSA: implications for comorbidities. Chest. 2015;147(1):266-274. doi:10.1378/chest.14-0500

4. Dempsey JA, Veasey SC, Morgan BJ, O’Donnell CP. Pathophysiology of sleep apnea. Physiol Rev. 2010;90(2):47-112. doi:10.1152/ physrev.00043.2008

5. Deniz M, Çiftçi Z, Ersözlü T, Gültekin E, Alp R. The evaluation of auditory system in obstructive sleep apnea syndrome (OSAS) patients. Am J Otolaryngol. 2016;37(4):299-303. doi:10.1016/j. amjoto.2016.03.004

6. Chung S, Yoon IY, Lee CH, Kim JW. The association of nocturnal hypoxemia with arterial stiffness and endothelial dysfunction in male patients with obstructive sleep apnea syndrome. Respiration. 2010;79 (5):363-369. doi:10.1159/000228905

7. el-Kady MA, Durrant JD, Tawfik S, Abdel-Ghany S, Moussa AM. Study of auditory function in patients with chronic obstructive pulmonary diseases. Hear Re. 2006;212(1-2):109-116. doi:10.1016/j. heares.2005.05.018
8. Chopra A, Jung M, Kaplan RC, et al. Sleep apnea is associated with hearing impairment: the Hispanic community health study/study of latinos. J Clin Sleep Med. 2016;12(5):719-726. doi:10.5664/jcsm.5804

9. Sardesai MG, Tan AK, Fitzpatrick M. Noise-induced hearing loss in snorers and their bed partners. J Otolaryngol. 2003;32(3):141-145. doi:10.2310/7070.2003.40256

10. Chandrasekhar SS, Tsai Do BS, Schwartz SR, et al. Clinical Practice Guideline: sudden Hearing Loss (Update). Otolaryngol Head Neck Surg. 2019;161(1_suppl):S1-S45. doi:10.1177/0194599819859885

11. Wu CS, Lin HC, Chao PZ. Sudden sensorineural hearing loss: evidence from Taiwan. Audiol Neurootol. 2006;11(3):151-156. doi: $10.1159 / 000091198$

12. Lionello M, Staffieri C, Breda S, et al. Uni- and multivariate models for investigating potential prognostic factors in idiopathic sudden sensorineural hearing loss. Eur Arch Otorhinolaryngol. 2015;272 (8):1899-1906. doi:10.1007/s00405-014-2992-8

13. Gozal D, Kheirandish-Gozal L. Cardiovascular morbidity in obstructive sleep apnea: oxidative stress, inflammation, and much more. $A m$ $J$ Respir Crit Care Med. 2008;177:369-375. doi:10.1164/rccm.2006 08-1190PP

14. Hwang JH, Chen JC, Hsu CJ, Liu TC. Association of obstructive sleep apnea and auditory dysfunctions in older subjects. Otolaryngol Head Neck Surg. 2011;144(1):114-119. doi:10.1177/0194599810390859

15. Vorlová T, Dlouhá O, Kemlink D, Šonka K. Decreased perception of high frequency sound in severe obstructive sleep apnea. Physiol Res. 2016;65(6):959-967. doi:10.33549/physiolres.933167

16. Sheu JJ, Wu CS, Lin HC. Association between obstructive sleep apnea and sudden sensorineural hearing loss: a population-based case-control study. Arch Otolaryngol Head Neck Surg. 2012;138 (1):55-59. doi:10.1001/archoto.2011.227

17. Kales A, Rechtschaffen A. A Manual of Standardized Terminology, Techniques and Scoring System for Sleep Stages of Human Subjects. Washington DC: Public Health Service, United States Government Printing Office; 1968.

18. Scoring EA. EEG arousals: scoring rules and examples: a preliminary report from the sleep disorders atlas task Force of the American Sleep Disorders Association. Sleep. 1992;15:173-184. doi:10.1093/sleep/ 15.2.173

19. Iber C, Ancoli-Israel S, Chesson A, Quan S. The AASM Manual for the Scoring of Sleep and Associated Events: Rules, Terminology and Technical Specifications. Westchester, IL: American Academy of Sleep Medicine; 2007.

20. Faul F, Erdfelder E, Buchner A, Lang AG. Statistical power analyses using $\mathrm{G}^{*}$ Power 3.1: tests for correlation and regression analyses. Behav Res Methods. 2009;41(4):1149-1160. doi:10.3758/BRM.41.4.1149

21. Kim JY, Ko I, Cho BJ, Kim DK. Association of obstructive sleep apnea with the risk of Ménière's disease and sudden sensorineural hearing loss: a study using data from the Korean national health insurance service. J Clin Sleep Med. 2019;15(9):1293-1301. doi: $10.5664 / \mathrm{jcsm} .7922$

22. Nakayama M, Masuda A, Ando KB, et al. A pilot study on the efficacy of continuous positive airway pressure on the manifestations of Ménière's disease in patients with concomitant obstructive sleep apnea syndrome. J Clin Sleep Med. 2015;11(10):1101-1107. doi: $10.5664 / \mathrm{jcsm} .5080$

23. Kim SK, Kim JH, Jeon SS, Hong SM. Relationship between sleep quality and dizziness. PLoS One. 2018;13(3):e0192705. doi:10.1371/ journal.pone. 0192705

24. Tsai MS, Lee LA, Tsai YT, et al. Sleep apnea and risk of vertigo: a nationwide population-based cohort study. Laryngoscope. 2018;128 (3):763-768. doi:10.1002/lary.26789

25. Fanfulla F, Grassi M, Taurino AE, D'Artavilla Lupo N, Trentin R. The relationship of daytime hypoxemia and nocturnal hypoxia in obstructive sleep apnea syndrome. Sleep. 2008;31(2):249-255. doi:10.1093/sleep/31.2.249 
26. Fischer Y, Yakinthou A, Mann WJ. Prevalence of obstructive sleep apnea syndrome (OSA) in patients with sudden hearing loss. a pilot study. HNO. 2003;51(6):462-466. doi:10.1007/s00106-002-0712-y

27. Lazarini PR, Camargo AC. Idiopathic sudden sensorineural hearing loss: etiopathogenic aspects. Braz. J Otorhinolaryngol. 2006;72(4):554-561.

28. Li X, Chen WJ, Zhang XY, et al. Inner ear function in patients with obstructive sleep apnea. Sleep Breath. 2020;24(1):65-69. doi:10.1007/s11325-019-01891-7

29. Seo YJ, Chung HJ, Park SY, et al. Lowest oxyhemoglobin saturation may be an independent factor influencing auditory function in severe obstructive sleep apnea. J Clin Sleep Med. 2016;12(5):653-658. doi:10.5664/jcsm.5786

30. Kayabasi S, Hizli O, Yildirim G. The association between obstructive sleep apnea and hearing loss: a cross-sectional analysis. Eur Arch Otorhinolaryngol. 2019;276(8):2215-2221. doi:10.1007/s00405-01905468-8
31. Capaccio P, Pignataro L, Gaini LM, et al. Unbalanced oxidative status in idiopathic sudden sensorineural hearing loss. Eur Arch Otorhinolaryngol. 2012;269(2):449-453. doi:10.1007/s00405-011-1671-2

32. Casale M, Vesperini E, Potena M, et al. Is obstructive sleep apnea syndrome a risk factor for auditory pathway? Sleep Breath. 2012;16 (2):413-417. doi:10.1007/s11325-011-0517-x

33. Huang YS, Chin WC, Guilleminault C, Chu KC, Lin CH, Li HY. Inflammatory factors: nonobese pediatric obstructive sleep apnea and adenotonsillectomy. J Clin Med. 2020;9(4):1028. doi:10.3390/ jem 9041028

\section{Publish your work in this journal}

Nature and Science of Sleep is an international, peer-reviewed, open access journal covering all aspects of sleep science and sleep medicine, including the neurophysiology and functions of sleep, the genetics of sleep, sleep and society, biological rhythms, dreaming, sleep disorders and therapy, and strategies to optimize healthy sleep.
The manuscript management system is completely online and includes a very quick and fair peer-review system, which is all easy to use. Visit http://www.dovepress.com/testimonials.php to read real quotes from published authors. 